\title{
Renewable electricity trading through SLAs
}

\author{
Georgios Methenitis ${ }^{1,2^{*}}$ (D) Michael Kaisers ${ }^{1}$ and Han La Poutré ${ }^{1,2}$
}

\author{
${ }^{*}$ Correspondence: \\ georgios.methenitis@cwi.nl \\ An extended abstract of this article \\ has been published under the title \\ "SLA-Mechanisms for Electricity \\ Trading under Volatile Supply and \\ Varying Criticality of Demand" \\ (Methenitis et al. 2017). An earlier \\ version of this article has also been \\ presented at the workshop \\ AMEC/TADA 2017, and may have \\ appeared in online repositories. \\ ${ }^{1}$ Centrum Wiskunde \& Informatica, \\ Amsterdam, The Netherlands \\ ${ }^{2}$ Delft University of Technology, \\ Delft, The Netherlands
}

\begin{abstract}
Energy systems are in transition towards more sustainable generation portfolios. In the envisioned smart grid generation will primarily depend on renewable power sources making uncertain quantities of electricity available, the delivery of which cannot be guaranteed. Current electricity tariffs promise certain delivery, and are thus not well-suited to trade uncertain quantities. However, if not traded the electricity might need to be curtailed, foregoing potential benefits for both supply and demand sides. We propose to adopt service level agreements (SLAs) that comprise quantity, reliability, and price, for electricity trading in settings where supply depends on volatile power sources. We define a characterization of the value degradation of tolerant and critical buyers with regards to the uncertainty of electricity delivery generalizing the widely used value of lost load (VoLL). This captures buyers' varying abilities to cope with uncertainty. We consider allocating SLAs to buyers using either a sequential second-price auction or the combinatorial Vickrey-Clarke-Groves (VCG) mechanism that is known to elicit truthful bids, and discuss the settings in which we can obtain truthfulness in the sequential setting. In addition, we empirically compare their performance and demonstrate that VCG dominates alternative allocations and vastly improves the efficiency of the proposed system, when compared to baseline allocations that only use the VoLL. This article hence contributes an essential component to the future smart grid by facilitating distributed energy trading under uncertainty.
\end{abstract}

Keywords: Electricity trading, Uncertainty, SLAs, VoLL, Mechanism design

\section{Introduction}

Energy systems are in transition towards more sustainable and distributed generation portfolios, where smaller scale producers and consumers will participate as autonomous agents in decentralized markets (Farhangi 2010). The main focus of this envisioned system is to maintain balance between available supply and demand. Maintaining balance becomes more challenging in face of generation from renewable resources such as the sun and wind, which are subjects to stochastic availability, and non-dispatchable. Their output cannot be regulated to match the demand, which is necessary to keep the system in balance. Therefore, demand side management (DSM) is necessary. DSM is the change in the behavior of the demand side, which can be enabled through, e.g., financial incentives (Strbac 2008; Palensky and Dietrich 2011). Dynamic pricing alongside scheduling of non-preemptive consumption loads are considered the main methodologies for

(c) The Author(s). 2018 Open Access This article is distributed under the terms of the Creative Commons Attribution 4.0 International License (http://creativecommons.org/licenses/by/4.0/), which permits unrestricted use, distribution, and reproduction in any medium, provided you give appropriate credit to the original author(s) and the source, provide a link to the Creative Commons license, and indicate if changes were made. 
balancing demand with uncertain supply. However, the former may introduce disruptive and unfavorable market behavior and thus planning and ahead prices are required (Braithwait et al. 2007), while the latter can violate the autonomy of consumer agents.

Service level agreements (SLAs) define agreements between service providers and service users, specifying the service and its characteristics (Verma 1999). SLAs can provide the contracting framework for balancing volatile supply with demand between buyers (e.g., customers participating in retail tariff schemes) and sellers of electricity (e.g., smallscale producers that base their generation portfolio on distributed energy resources). The characteristics of the service vary depending on the application, e.g., quantity, price of a service.

To our best knowledge, we first interpret SLAs as a direct extension of conventional electricity tariffs, which ensure delivery (100\% quality) and a fixed $\mathrm{kWh}$ price ( $0 \%$ risk). In contrast to the current straightforward contracts, SLAs can be extended to include more features (e.g., delivery time, reliability, penalty for no delivery).

The process of specifying and allocating SLAs to buyers participating in the electricity market can be structured as a mechanism (Nisan and Ronen 2001; Hurwicz and Reiter 2006). The design of the mechanism depends on the structure of the SLAs and the features they comprise. We specifically study SLAs comprising the following features:

Quantity The quantity of electricity that is subject to be transferred from the service provider to the service user.

Reliability The probability of successful delivery of the quantity of electricity that is specified in the SLA.

Price The price per unit of the transferred quantity.

The features described above provide a basic SLA contracting framework for electricity trading between buyers and sellers in settings where the availability of electricity is not guaranteed, and buyers' ability to cope with uncertainty vary.

We proceed to illustrate the concepts discussed so far in the following example:

Example 1 A seller holds a prediction of its generation for the next day during $1 \mathrm{pm}-2 \mathrm{pm}$. The generation is not certain; there is 90\% probability that the seller generates 1 unit and $50 \%$ probability 2 units of electricity. There are two buyer agents, both have unit demand. Let us now assume that the one buyer is a hospital that needs to perform a task, and the other buyer is an electric vehicle (EV) that needs to charge its battery. Assuming that there is no other seller in the system we consider, the two agents can agree on SLAs of $90 \%$ or $50 \%$ reliability with the seller for the unit demand they require. Considering that the importance of the task the hospital needs to complete is higher than the EV's, it is socially optimal to assign the most certain unit of generation to the hospital.

As illustrated in Example 1, it is socially desirable that reliable electricity is allocated to critical demand, and the risk of load-shedding is assigned to less critical buyers that in turn perform this task at lower social cost.

The widest adopted concept to measure criticality in the literature as well as in practice is the value of lost load (VoLL) (Kariuki and Allan 1996). The VoLL is defined as the estimated amount that customers receiving electricity through contracts would be willing 
to pay to avoid a disruption in their electricity service. Revisiting Example 1, we can safely say that, the VoLL is higher for the hospital than for the EV.

In this paper, we study the problem of allocating SLAs (efficiently) for electricity trading. We assume uncertain electricity generation with a known distribution, and buyers with different preferences with regards to the uncertainty of being served. The main contributions of this work can be summarized as follows:

- We define a contracting framework through SLAs that enables energy trading under uncertain supply (“Contracting framework” section).

- We propose a family of exponential functions that characterizes the buyers' varying degrees of criticality, thus generalizing the value of lost load with costs associated to the risk of failed delivery ("Critical \& tolerant buyers" section).

- We apply two mechanisms to assign SLAs to agents of different types, and incentivize truthfulness for strategic buyer agents ("Auction-based SLA allocation" section).

- Last, we show that the efficiency of the proposed system vastly improves in face of buyers with varying abilities to cope with uncertainty ("Evaluation \& discussion" section).

The rest of the paper is structured as follows: "Related work" section provides an overview of the literature that is relevant to our work. "Uncertainty in the smart grid" section formulates the problem of purchasing electricity in the presence of uncertain supply. In "Contracting framework" section, we introduce the structure of the proposed framework through SLAs, we further define the representation of the uncertain supply, and last, we propose a value function that determines different types and preferences of buyers. In "Auction-based SLA allocation" section, we provide mechanisms to assign SLAs to buyers, and we examine incentive compatibility issues arising in the domain. In "Evaluation \& discussion" section, we evaluate our proposed setting through simulation. Last, "Conclusion" section concludes this paper proposing interesting research directions of this work.

\section{Related work}

Several recent works have studied the problem of uncertainty in the smart grid. Renewable electricity generation comes at the cost of increased volatility in such systems, which is the main reason for the potentially high costs associated with balancing supply and demand through conventional generation. However, incentives for balancing can be forwarded to the demand-side through demand-response allocation mechanisms (Ma et al. 2016), prediction of use tariffs (Vinyals et al. 2014), or tariffs with variable risk for the consumers who can alleviate balancing costs from the retailer side (Methenitis et al. 2016). Other works focus on the planning optimization of flexible consumers of electricity that can yield their own incentives (utility maximization) in the uncertain environment of the smart grid (Angelidakis and Chalkiadakis 2015; Gerding et al. 2016), or on online mechanisms for local matching of flexible demand and uncertain supply from renewable generation (Ströhle and Flath 2016).

In line with our assumptions, the problem of balancing volatile supply with flexible demand has been studied in recent works. In the presence of delay tolerant customers, service delays can be minimized via the Lyapunov optimization technique without the requirement of a-priory knowledge of the underlying statistics (Neely et al. 2010). 
Furthermore, to deal with uncertain supply, potential scenarios of future renewable supply can be considered in Monte-Carlo planning. Based on the likelihood of scenarios, which is updated whenever new information about the supply is becoming known, an online mechanism that uses the concept of pre-commitment by the demand side has been proposed to allocate the available supply to flexible demand in order to maximize social welfare (Ströhle et al. 2014). Similarly, the problem of matching uncertain supply with demand is considered as a multi-agent sequential decision making problem, where beliefs over states of the system are replaced with beliefs over future supply scenarios (Walraven and Spaan 2015). Each of the works above has a notion of cost (or criticality similar to VoLL), but none of those considers varying risk premiums for uncertainty.

SLAs have been considered as a tool for monitoring and coordination to ensure trustworthiness between different stakeholders, primarily with regards to the business processes, to ensure trustworthiness between different stakeholders (Gustavsson et al. 2011; Hussain et al. 2012), or as a negotiation protocol (Amato et al. 2014). In contrast to our work, the discussion remains conceptual, and no quantitative implications on costs or efficiency are given. SLAs have also been used in resource allocation in computational grids to ensure the optimal allocation of computational resources and fair satisfaction of the participants through negotiations (Silaghi et al. 2012). Here, the embedding is not in the energy domain, and the focus of the work is on strategic negotiation rather than the elicitation of truthful reports. Another related work has studied task allocation market mechanisms for multiple suppliers of finite or uncertain capacity (Dash et al. 2007).

Similarly to product (service) differentiation in power systems engineering and energy markets (Oren and Smith 1993; Salah et al. 2017), we propose SLAs that differentiate in the reliability of successful delivery. Product differentiation in electricity trading also includes but is not limited to: (i) deadline-differentiated trading, where consumers agree to defer the service of pre-determined loads in exchange for lower prices (Bitar and $\mathrm{Xu}$ 2017), (ii) varying probabilities of electricity service interruptions (Chao et al. 1986), (iii) reliability differentiation for trading spinning reserve capacity (Siddiqi and Baughman 1995). Our work is more related with the mechanism design approach proposed in (Bitar and $\mathrm{Xu}$ 2017). However, here we consider buyers that have different valuations with regard to the probability of successful delivery of electricity service. Other outlined research studies optimal pricing policies and not the design of mechanisms to elicit truthful reports from buyers (Chao et al. 1986; Siddiqi and Baughman 1995).

In the closest state of the art work, the authors study the viability of selling uncertain quantities of wind generation with variable-reliability (Bitar et al. 2012). They further explore the connection between uncertainty in the generation and the costs for reserve capacity, and real-time markets. We follow a similar idea, but we focus our attention on the characterization of the demand with respect to its criticality, as well as the design of the mechanisms to assign demand through SLAs for electricity trading to strategic buyers with different preferences.

To the best of our knowledge we are providing the first work that adapts SLAs for energy trading under uncertain supply, providing both a discussion of the buyers' incentives and an empirical evaluation in illustrative settings. Here, SLAs are the resulting contracts that are allocated through mechanisms, to distribute uncertain supply to buyers of different types. 


\section{Uncertainty in the smart grid}

The Smart Grid is an electricity grid innovation that emphasizes the transition from the traditional paradigm of passive distribution and consumption towards an energy network in which each node may take on an active role. This is exemplified by the increasing adoption of renewable generation (primarily from solar and wind) that makes households prosumers, serving some of their own load or even producing excess generation. The stochastic variation in generation introduces uncertainty in the supply. In addition, active control of loads such as heat pumps and batteries or charging controllers for electric vehicles introduce flexibilities - yet in the absence of clear incentives these flexible loads may be notoriously difficult to forecast.

In the proposed SLA allocation mechanism, participating buyer agents (i.e., electricity customers) purchase quantities of electricity through contracts of a specific quantity, reliability, and price, subject to uncertain supply and its availability. In our setting, we consider only one seller and no outside option for the buyers. Let $s$ denote the seller and $\mathcal{B}$ the set of buyer agents, there are $n$ buyer agents in the set $\mathcal{B}$, such that $\mathcal{B}=\{1, \ldots, n\}$.

Large volumes of electricity in Europe are traded in the day-ahead markets, where a clearing price is the result of double auctions between buyers and sellers who are placing their bids for each 1-hour time interval of the next day. Our two-step model may serve as a fundamental model of the day-ahead auction process in current electricity markets, but could also be applied at different horizons: In the first step, ahead timestep, the mechanism holds a prediction (i.e., probability distribution function) of the available supply for the realization timestep. The mechanisms outlined in later sections are thus also applicable for shorter horizons, e.g., hour(s) ahead, as they may arise in local microgrids with flexible power-to-heat demand.

Let $Q$ denote the random variable of the prediction of supply at the timestep ahead, $q \in \mathbb{R}^{+}$is the observed realization of supply at the realization timestep. We further denote the cumulative density function of the random variable $Q$ with $F_{Q}(q)=P(Q \leq q)=$ $\int_{0}^{q} f_{Q}(x) d x$, where $f_{Q}(x)$ is the probability density function. Similarly, each buyer agent $i$ from the set $\mathcal{B}$ has a demand for electricity $d_{i}$, which we assume is fixed and known by the agent ahead of time, considering hardware assets that induce a deterministic demand.

Buyer agents, based on their preferences, can get assigned SLAs of certain quantity, reliability and price, ensuring that their demand will be satisfied with some probability in the realization timestep. The observed realization $q$ of the electricity generation determines how much load can be served, and the mechanism determines the set of buyers that are indeed served such that the SLAs are satisfied in expectation with regards to their reliability.

\section{Contracting framework}

In this section we focus on modeling the contracting framework where SLAs can be assigned to buyers in the presence of uncertain generation. We further define the basic structure of the SLAs, and subsequently the characterization of buyers' preferences with regards to the reliability of the SLAs.

\section{Service level agreements}

As outlined in "Introduction" section, an SLA is a triplet $\left(d_{i}, \gamma_{i}, p_{i}\right)$, which comprises the quantity $d_{i}$, the reliability $\gamma_{i}$, and the price $p_{i}$ per transferred unit of electricity for 
the buyer agent $i \in \mathcal{B}$. For the remainder of this paper we assume unit-demand buyers, $d_{i}=1, \forall i \in \mathcal{B}$. We further assume that the delivery of the electricity of the assigned SLAs is either successful or not. Let $\hat{d}_{i}=\left\{0, d_{i}\right\}$ denote the transferred quantity to the buyer agent $i$. We define $v_{i}$ as the marginal value that the successful delivery of electricity brings to the buyer agent, $v_{i}=\alpha_{i} \hat{d}_{i}, \alpha_{i} \in \mathbb{R}^{+}$. The value of $\alpha_{i}$ refers to the private value of the agent $i$ when delivery is assured $\left(\gamma_{i}=1\right)$, or equivalently the VoLL. Considering the binary model for the value that the transferred quantity of electricity delivers to the buyer agent, the value can be either fully obtained or not $\left(v_{i}=\left\{0, \alpha_{i} d_{i}\right\}\right)$. The expected value of the buyer, given the demand $d_{i}$ and the reliability $\gamma_{i}$, is equal to:

$$
\nu_{i}\left(\gamma_{i}\right)=\alpha_{i} d_{i} \gamma_{i} .
$$

Let $S_{Q}(q)$ denote the reliability function (also known as survival function) of the seller agent $s$. Figure 1 illustrates $S_{Q}(q)$. Note that $S_{Q}(q)=1-F_{Q}(q)$, where $F_{Q}(q)$ is the cumulative density function of the random variable $Q$. The reliability function $S_{Q}(q)$ determines the probability that the generation exceeds a certain value $q$. The dotted area represents an SLA (no price $p_{i}$ is determined here) between the seller agent and the buyer agent $i$. The demand of the buyer agent $i$ is equal to $d_{i}$ and the reliability of the specific SLA is $\gamma_{i}=$ $S_{Q}\left(w+d_{i}\right)$, where $w$ is the demand that is already deducted by previously allocated SLAs.

Given the assumption of unit-demand buyers, we further consider that the demand quantity $d_{i}$ of a buyer agent is not comparable to the total demand of the $n$ agents and the expected generation of the seller agent, such that the following holds: $S_{Q}(w) \approx S_{Q}\left(w+d_{i}\right)$ and $P\left(w \leq q \leq w+d_{i}\right) \approx 0$. The probability of partial delivery of electricity approximates zero in the unit-demand case, justifying the binary model we use for the value that the successful delivery brings to the buyer.

We proceed to show that buyers may have different preferences with regards to the probability of being served. Hence, buyers' utility function depends on the reliability $\gamma$ of SLAs.

\section{Critical \& tolerant buyers}

The expected value of a buyer in Eq. 1 is linearly dependent on the reliability $\gamma_{i}$ of the SLA. Since the system gives raise to risk, we can distinguish between different attitudes

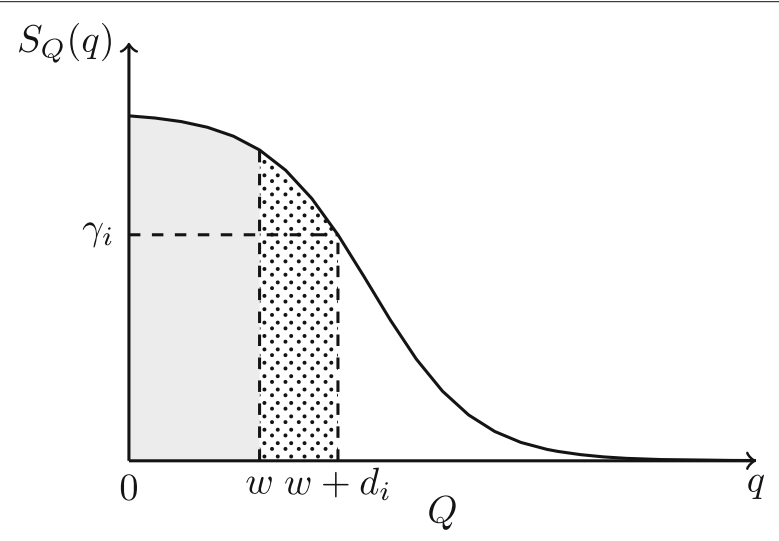

Fig. 1 The reliability function of the seller. The thick line illustrates the reliability function $S_{Q}(q)=P(Q>q)$ of the random variable $Q$. The dotted area represents the portion of demand $d_{i}$ of the buyer agent $i$ with reliability $\gamma_{i}=S_{Q}\left(w+d_{i}\right)$. The gray shaded area represents already assigned SLAs between the seller and the buyer agents 
of buyers towards risk, from critical to tolerant, as it is usual in economics and expected utility theory (Ingersoll 1987). We define the generalized expected value function $\mathcal{V}_{i}\left(\gamma_{i}\right)$, where the reliability $\gamma_{i}$ induces the risk in the form of uncertain delivery of the specified in the SLA quantity:

$$
\mathcal{V}_{i}\left(\gamma_{i}\right)=\alpha_{i} d_{i} u_{i}\left(\gamma_{i}\right)
$$

where $u_{i}\left(\gamma_{i}\right)$ encompasses the attitude of the buyer to the reliability $\gamma_{i}$. Note that for $u_{i}\left(\gamma_{i}\right)=\gamma_{i}, \mathcal{V}_{i}\left(\gamma_{i}\right)$ becomes equal to $v_{i}\left(\gamma_{i}\right)$ in Eq. 1 . The generalized expected value function in Eq. 2 should embrace some common sense properties:

- Buyers have zero value for no reliability, i.e., $\mathcal{V}_{i}(0)=0, \forall i \in \mathcal{B}$.

- Buyers have maximum value for no uncertainty, i.e., $\mathcal{V}_{i}(1)=\alpha_{i} d_{i}, \forall i \in \mathcal{B}$.

- Buyers have higher value for more certainty in the delivery, i.e., $\mathcal{V}_{i}\left(\gamma_{i}\right) \geq \mathcal{V}_{i}\left(\gamma_{i}-\varepsilon\right), \forall \varepsilon \in \mathbb{R}^{+}, \forall i \in \mathcal{B}$ (monotonicity).

- Buyers have positive value for any positive reliability, i.e., $\mathcal{V}_{i}\left(\gamma_{i}\right)>0, \forall \gamma_{i}>0, \forall i \in \mathcal{B}$ (buyers' willingness to participate).

Here, we consider a variation of the exponential utility function (Ingersoll 1987). In line with the aforementioned properties of $\mathcal{V}\left(\gamma_{i}\right)$, we define $u_{i}\left(\gamma_{i}\right)$ with regards to $\beta_{i} \in \mathbb{R}$.

$$
u_{i}\left(\gamma_{i}\right)= \begin{cases}\frac{1-e^{-\beta_{i} \gamma_{i}}}{1-e^{-\beta_{i}}}, & \beta_{i} \neq 0 \\ \gamma_{i} & , \beta_{i}=0\end{cases}
$$

This variation of the exponential utility function in Eq. 3 can be substituted into Eq. 2, yielding the expected value of the buyer agent with regards to the reliability $\gamma_{i}$, where $\beta_{i} \in \mathbb{R}$ distinguishes the buyer type from critical $\left(\beta_{i}<0\right)$ to tolerant $\left(\beta_{i}>0\right)$. For $\beta_{i}=0$ the expected value function $\mathcal{V}_{i}\left(\gamma_{i}\right)$ becomes equal to the expected value in Eq. 1. Figure 2 illustrates the function $\mathcal{V}_{i}\left(\gamma_{i}\right)$ for different values of $\beta_{i}$.

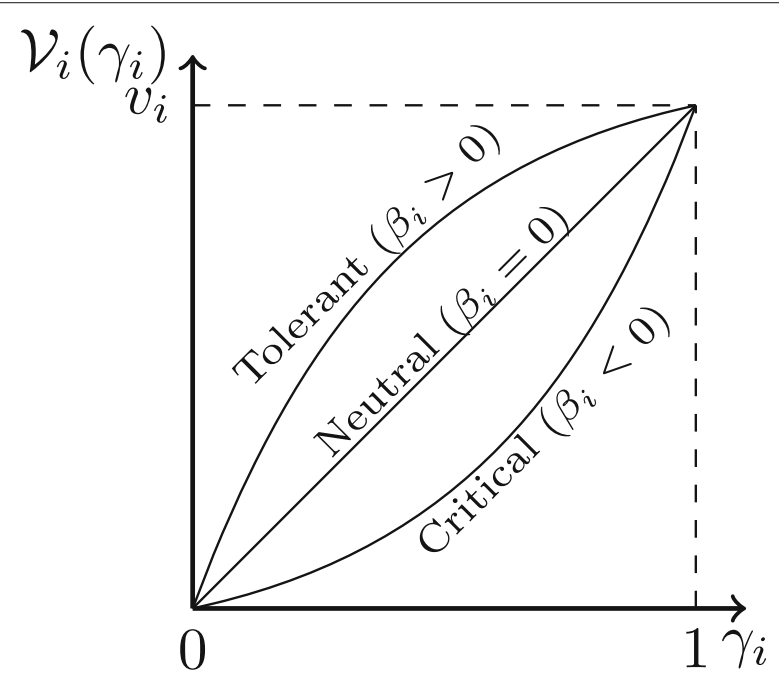

Fig. 2 The generalized expected value function of buyers. The expected value function $\mathcal{V}_{i}$ of a buyer agent with regards to the reliability $\gamma_{i}$ of an SLA. At full reliability $\left(\gamma_{i}=1\right)$ the expected value of the SLA by the buyer agent is equal to $v_{i}$ which is determined by the private value $\alpha_{i}$ and the demand $d_{i}$. The value of $\beta$ distinguishes different attitudes of buyer $i$ towards the reliability $\gamma_{i}$. The values used for the illustration are $\beta_{i}=\{-2.5,0,2.5\}$ 
Given the above definition of the generalized expected value function, we can categorize buyer agents with regards to their attitudes towards reliability:

Critical For $\beta_{i}<0$, the expected value function is convex, representing a critical (riskaverse) buyer. There is a stiff degradation of the value with regards to the uncertainty of the delivery for the critical buyer, resulting from opportunity costs that arise in case of failed delivery.

Tolerant For $\beta_{i}>0$, the expected value function is concave, the buyer is tolerant (riskseeking). Lower reliability translates to a rather high expected value, resulting from opportunity value that arises in case of failed delivery.

Neutral For $\beta_{i}=0$, the expected value is linearly dependent to the reliability as in Eq. 1, representing a neutral buyer.

The generalized expected value function outlines a realistic model for capturing buyers' preferences. The proposed function describes the graceful or stiff degradation of the VoLL with regards to the probability of successful delivery. The type of the buyer agent $i$ is characterized by the tuple $\left(\alpha_{i}, \beta_{i}\right)$. In the context of electricity markets, the same quantity of electricity may have different value for different consumers, which is captured in our model by $\alpha_{i}$. Furthermore, the incurred value of a lost load with regards to the probability of electricity delivery is determined in our model by $\beta_{i}$. The function $\mathcal{V}_{i}\left(\gamma_{i}\right)$ indicates the VoLL of a buyer agent given the reliability of the SLA as follows: $V o L L=\mathcal{V}_{i}(1)-\mathcal{V}_{i}\left(\gamma_{i}\right)$. The generalized expected value function can be defined in both one-shot and repeated settings, where in the latter agents could vary their types with regards to the outcome of their earlier assignments.

Let us now proceed with an example of a realistic scenario where the value of a buyer agent depends on the probability of being served, and thus the opportunity cost or value that arises in case of failed delivery.

Example 2 An EV-taxi is operating its task (transporting people) with a half-full battery capacity with no urgent need to charge its battery. The EV-taxi can represent a tolerant buyer since there is value that is gained in case of charging (future benefits), and opportunity value that is gained in case of not charging (immediate benefits resulting by not pausing its task). When the capacity of its battery is running low, the EV-taxi can better be represented by a critical buyer since the value in case of no delivery (immediate benefits for task continuation) is decreasing relatively to the value that can be gained by charging and pausing its task.

Given the above example, the generalized value function can distinguish buyers in terms of opportunity cost or value that could be incurred or gained respectively with regards to the probability of successful delivery of electricity.

The price $p_{i}$ that is specified in the SLA, and hence the expected utility of the buyer agent $i$ is determined by the resultant allocation of the mechanism (see "Auction-based SLA allocation" section). Let $\mathcal{U}_{i}$ denote the expected utility of buyer agent $i$,

$$
\mathcal{U}_{i}=\mathcal{V}_{i}\left(\gamma_{i}\right)-d_{i} p_{i} \gamma_{i}
$$

where the expected payment that is transferred from the buyer to the seller upon delivery is subtracted from the expected value. 
In the section that follows we discuss how SLAs can be allocated to buyers with different private values for SLAs of varying reliability.

\section{Auction-based SLA allocation}

Auctions are widely used in competitive electricity markets that take place day-ahead (Contreras et al.2001), and they are known to yield efficient allocations even in cases there is uncertainty about buyers' valuations for items to be sold (Krishna 2010). We consider auctions as the method to allocate SLAs among buyers with varying private types, with regards to the value of their demand and their flexibility under the presence of uncertainty in the electricity market. Here, we assume no agency for the seller, who only serves as the mechanism to allocate SLAs to the buyers.

Let $A$ be an allocation from the set of all feasible allocations $\mathcal{A}, A \in \mathcal{A}$, as the triplet of vectors $(\mathbf{d}, \boldsymbol{\gamma}, \mathbf{p})$, where each entry $A^{(i)}=\left(d_{i}, \gamma_{i}, p_{i}\right)$ is an allocated SLA between the seller agent $s$ and buyer agent $i$. Considering unit-demand buyers, $d=d_{i}=1, \forall i \in \mathcal{B}$, an allocation can be written as $A=\left\{o_{1}, o_{2}, \ldots, o_{n}\right\}$, where $o_{i} \in \mathbb{Z}_{1}^{n}$ denotes the order of an allocated SLA. Given the order $o_{i}$ of the SLA $\left(d_{i}, \gamma_{i}, p_{i}\right)$ the reliability $\gamma_{i}$ is given by: $\gamma_{i}=S\left(o_{i} d\right)$ (see Fig. 1). Following the definition of the reliability function, $\forall j \in \mathcal{B}$, $\gamma_{i} \geq \gamma_{j}$, where $o_{i}<o_{j}$, the reliability is monotonically decreasing with the order. The set of feasible allocations $\mathcal{A}$ includes all allocations $A$ for which every element appears only once in the set. The value of an SLA by the buyer agent $i$ is determined by Eq. 2 with regards to the reliability $\gamma_{i}$ and consequently the order $o_{i}$. We define buyers' expected social value as the sum of the expected values of the set of the buyer agents given the allocated SLAs as follows: $\sum_{i \in \mathcal{B}} \mathcal{V}_{i}\left(\gamma_{i}\right)$, where $\gamma_{i}=S\left(o_{i} d\right)$ is determined according to $A$. The allocation $A$ further determines the order that buyers get served, for each buyer $\forall i \in \mathcal{B}$, served $_{i}=\left(q \geq o_{i} d\right)$, which follows from $P\left(\right.$ served $\left._{i}\right)=\gamma_{i}$.

\section{Sequential second-price auction}

First, we consider a sequential second-price auction (SSPA) (Leme et al. 2012; Syrgkanis and Tardos 2012), as an SLA allocation mechanism for all supply that may become available at the realization timestep. Items, SLAs in this case, are auctioned off one at a time. Given the assumption of unit-demand buyers, the seller auctions off SLAs of quantity $d$. We consider that the seller starts auctioning SLAs of decreasing reliability, such that the first SLA has reliability of $S(d)$, the second $S(2 d)$, and so on. The order that items are auctioned off in sequential auctions affects the auctioneer's revenue (Elkind and Fatima 2007). Given the monotonicity property of the generalized value function in Eq. 2, SSPA of decreasing reliability SLAs maximizes the revenue of the seller. In later sections of this paper we also evaluate the case where the seller auctions off SLAs of increasing reliability.

A second-price auction, also known as a Vickrey auction (Vickrey 1961), is held by the seller in every round $k$ of the auction where an SLA of reliability $S(k d)$ is auctioned off. Let $\mathcal{V}_{i}^{\prime}(S(k d))$ denote the reported value of the buyer agent $i$ with regards to the reliability $S(k d)$ offered in the $k$-th round of the sequential auction. Each buyer $i$ places a sealed bid $z_{i}$, which is equal to the reported value with regards to the reliability $S(k d)$. The winner agent $w \in \mathcal{B}$ is determined as the buyer who submits the highest bid, $w=\operatorname{argmax}_{i} z_{i}$, while the winning agent pays to the seller the price $p_{w}$ of the second 
highest bid, such that $p_{w}=\max _{i \neq w} z_{i}$. The winner agent $w$ of every round $k$ of the sequential auction is allocated an SLA of unit quantity $d$, reliability $S(k d)$, and price $p_{w}$. The winner $w$ participates no further in the next rounds of the auction. In each round of the auction only the winner agent $w$ knows the price of the assigned SLA, i.e., we assume no price-discovery. In the second-price auction a buyer cannot increase its probability of allocation by increasing its bid in case the second highest bid is lower. In the opposite case, the buyer could win the auction by increasing its bid, however, this would result in negative utility. Here, we assume that buyers do not bid higher than their value, i.e., no over-bidding.

We proceed to establish the settings of the second-price auction with the following assumption.

Assumption 1 We assume settings where strategic buyers do not communicate their preferences to other participating agents, and there is no knowledge regarding the number and the distribution of the buyers participating in the auction. Furthermore, no buyer knows the reliability function of the seller, buyers only know that the reliability of the next SLA to be auctioned off is lower or equal to the reliability of the SLA that is being auctioned. The reliability function $S$ is defined for all demand quantities. However, not all demand is guaranteed to be satisfied within an SSPA, since SLAs may be assigned with zero reliability.

Theorem 1 Given Assumption 1, each round of the SSPA is an isolated Vickrey auction and therefore the mechanism is dominant-strategy incentive-compatible (DSIC).

Proof Each round of SSPA can be the last round or the round before with value arbitrarily close to zero and therefore can be treated as an isolated Vickrey auction (Vickrey 1961). Buyers' dominant strategy is to report their true value function, i.e., $\mathcal{V}_{i}^{\prime}(S(k d))=\mathcal{V}_{i}(S(k d)), \forall i \in \mathcal{B}$.

The Proof of Theorem 1 exploits the property that no stochastic model can be built by the buyer regarding follow up rounds of SSPA. Given Assumption 1, each round in which the buyer can wait without participating (bidding low or zero), does not add any information regarding the distribution of future bids of other agents. Consequently, there is no stochastic model which can compute an expectation of future utilities in case of waiting the next round to bid truthfully. To prove Theorem 1, we assume that the buyer is deterministic choosing to participate as this was the last round of the auction to maximize the likelihood of getting assigned an SLA of positive reliability.

SSPA mechanism auctions off SLAs of decreasing reliability to buyers in a sequential fashion. We showed that given the assumptions of the proposed SSPA mechanism, there is no incentive for a strategic buyer to misreport its value function, and therefore the mechanism elicits truthful reports.

\section{Vickrey-Clarke-Groves mechanism}

Sequential second-price auctions can be suitable mechanisms to allocate SLAs to buyer agents, however the allocation of the SLAs depends more on the value $\alpha$, and less on the criticality of the buyer, $\beta$. Consider the following example: 
Example 3 There are two unit-demand buyers, agent 1 valuates $90 \%$ of successful delivery $\mathcal{V}_{1}(90 \%)=a$ and $\mathcal{V}_{1}(50 \%)=3 / 4 a$ and agent $2, \mathcal{V}_{2}(90 \%)=a / 2, \mathcal{V}_{2}(50 \%) \approx 0$, using the sequential auction proposed in "Sequential second-price auction" section, agent 1 is assigned the SLA with 90\% while agent 2 is assigned 50\%. Assuming zero payments, the resulting social value of the above assignment is a. However, the socially optimal allocation would be agent 2 to be assigned $90 \%$ and agent 1 with $50 \%$ resulting in social value of $5 / 4$ a.

Similarly to the above example, in Fig. 3 (top) we illustrate the expected value functions of three buyers of diverse types. In Fig. 3, middle and bottom bar charts show two different allocations alongside the assigned reliability and the corresponding expected value. The dashed line presents the reliability of the allocated SLAs. In the greedy allocation (middle), each slot is assigned to the buyer agent who has the highest bid (SSPA). The allocation that yields the optimal social value is illustrated in the bottom figure. SSPA myopically allocates the highest bidder in each round (with no regards to the social value loss) and thus results in a suboptimal social value.
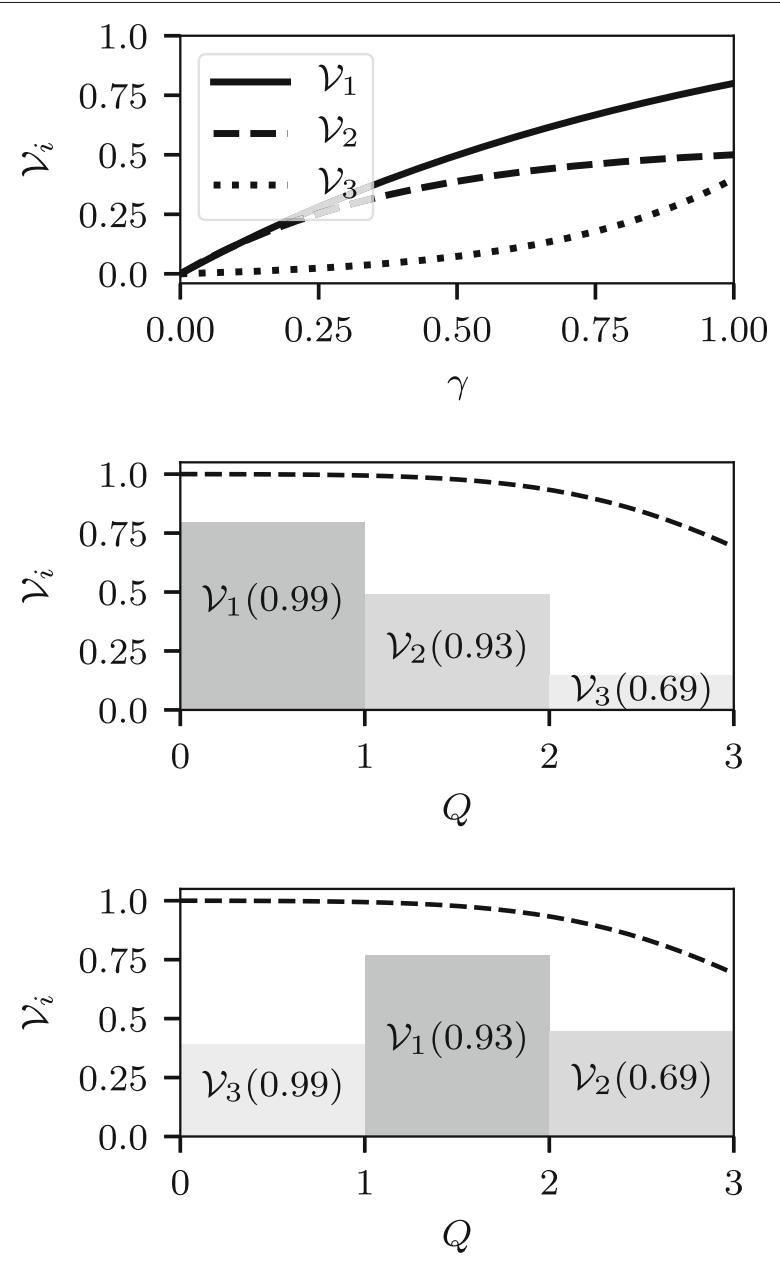

Fig. 3 Myopic and optimal SLA allocation. The value functions $\mathcal{V}_{i}$ of the buyer agents with regards to the reliability $\gamma$ of the possible SLAs (top). Resulting expected values of the buyer agents in the sequential second-price auction (middle). Resulting expected values of the buyer agents in VCG mechanism (bottom). The dashed line illustrates the reliability over allocated SLAs 
Combinatorial auctions are means to derive socially optimal allocations (Cramton et al. 2007). In a combinatorial auction all buyers submit their bids for the whole bundle of items and the auctioneer computes the optimal allocation, which maximizes the social welfare. We consider Vickrey-Clarke-Groves (VCG) (Nisan et al. 2007). Given the vector $\gamma$ of decreasing reliability of the available SLAs such that $\gamma=\langle S(d), S(2 d), \ldots, S(n d)\rangle$, each buyer submits a vector of bids $\mathbf{z}_{i}=\left\langle\mathcal{V}_{i}^{\prime}(S(d)), \mathcal{V}_{i}^{\prime}(S(2 d)), \ldots, \mathcal{V}_{i}^{\prime}(S(n d))\right\rangle$, which includes the reported value of buyer $i$ for each available reliability. Recall that $A \in \mathcal{A}$ is a feasible allocation $A=\left\{o_{1}, o_{2}, \ldots, o_{n}\right\}$, where $o_{i}$ denotes the order over the decreasing reliability SLAs of the allocated agent. We further define $A^{-i} \in \mathcal{A}^{-i}$ as a feasible allocation of all buyer agents excluding agent $i$. Let $A_{\text {opt }}$ denote the optimal allocation such that $A_{\text {opt }}=\operatorname{argmax}_{A \in \mathcal{A}} \sum_{i \in \mathcal{B}} \mathcal{V}_{i}(A)$.

In VCG, the price $p_{i}$ of each buyer agent is determined by its marginal contribution, which is equal to:

$$
p_{i}=\sum_{j \in \mathcal{B} \backslash i} \mathcal{V}_{j}\left(A_{\text {opt }}^{-i}\right)-\sum_{j \in \mathcal{B} \backslash i} \mathcal{V}_{j}\left(A_{\text {opt }}\right)
$$

where $A_{\text {opt }}^{-i}$ is the optimal allocation without agent $i$ present. The price $p_{i}$ is determined by the difference between the optimal social value that is achieved when agent $i$ is not part of the set $\mathcal{B}$ and the sum of the values that all agents excluding agent $i$ achieve under the optimal allocation $A_{\text {opt }}$ including agent $i$. Hence, each agent pays the loss incurred to the society by its presence. In VCG mechanism, it is a dominant strategy for buyers to report their valuations truthfully (Nisan et al. 2007).

In most combinatorial problems, computing an optimal allocation lies in the class of NP-complete problems, making these intractable even for few participants. In what follows, we show that the SLA allocation problem can be solved optimally in polynomial time.

Corollary 1 The problem of SLA allocation among the unit-demand buyer agents can be solved optimally in polynomial time $\mathcal{O}\left(n^{3}\right)$.

Proof The unit-demand SLA allocation problem is equivalent to the linear assignment problem (LAP), where $n$ agents have to be assigned $n$ tasks while the number of tasks is equal to the number of agents. Each task stands for a slot in allocation $A \in \mathcal{A}$. It can be solved optimally in polynomial time, $\mathcal{O}\left(n^{3}\right)$, by the Hungarian method (Kuhn 1955).

Corollary 1 shows that buyers can be assigned SLAs that maximize the social welfare in polynomial time (i.e., $\mathcal{O}\left(n^{3}\right)$ ). Furthermore, there exist implementations that can speed up further the computation of the optimal assignment in LAPs (Date and Nagi 2016). This makes the VCG mechanism a tractable solution for practical applications of our model.

\section{Evaluation \& discussion}

In this section we evaluate the performance of the studied mechanisms to allocate SLAs to buyers of different types. Specifically, we evaluate: the VCG mechanism (see "Vickrey-Clarke-Groves mechanism" section), SSPA where the seller auctions off SLAs of decreasing reliability (SPD) as described in "Sequential second-price auction" section, and increasing reliability (SPI). 
To study the efficiency of the proposed system when compared to a baseline allocation considering only the value of lost load (VoLL), we further compare against two mechanisms where only the value of buyers for certain delivery $\left(\mathcal{V}_{i}(1)=\alpha_{i} d_{i}\right)$ is used for the allocation. In both mechanisms, a simultaneous second-price auction is used for SLAs of certain delivery $(\gamma=1)$. In the first baseline mechanism (POB), the value function in Eq. 1 is used for the buyers, and thus we consider only neutral buyers (i.e., no added value or cost is generated as a result of the uncertainty). In the second baseline mechanism (POC), the generalized value function in Eq. 2 is used.

We evaluate and compare all the aforementioned mechanisms with regards to the social value, and the social welfare. For the remainder of this section, social value (SV) is defined as the average value of buyers. Following Eq. 2,

$$
\mathrm{SV}=\frac{1}{|\mathcal{B}|} \sum_{i \in \mathcal{B}} \mathcal{V}_{i}\left(\gamma_{i}\right)
$$

Social welfare $(\mathrm{SW})$ is the average of the expected utilities of the buyers, from Eq. 4,

$$
S W=\frac{1}{|\mathcal{B}|} \sum_{i \in \mathcal{B}} \mathcal{U}_{i}
$$

Consequently, the expected seller's surplus can be written as $\mathcal{U}_{s}=\sum_{i \in \mathcal{B}} d_{i} \gamma_{i} p_{i}=$ $|\mathcal{B}|(S V-S W)$, which is equal to the expected payments from the buyers to the mechanism (seller).

\section{Diversity in the criticality}

First, we analyze the influence of diversity in the criticality $\beta$ of the buyers to the social value. Recall that $\alpha$ determines the value of the buyer for reliability equal to one, and $\beta$ characterizes the buyer in term of its criticality to the uncertainty of the delivery. We consider settings where buyers have similar private values $\alpha$ for electricity usage $\forall i \in$ $\mathcal{B}, \alpha_{i} \sim \mathcal{U}(0.5,1.0)$, which realistically captures buyers with similar needs and valuations for electricity (e.g., households). The random variable of the supply is normally distributed $Q \sim \mathcal{N}\left(\mu_{Q}=20, \sigma_{Q}=5\right)$, while the total demand exceeds by $20 \%$ the expected supply, $\sum_{i \in \mathcal{B}} d_{i}=1.2 \mu_{Q}=|\mathcal{B}|$ (24 buyers). We consider that $\forall i \in \mathcal{B}, \beta_{i} \sim \mathcal{U}(-D, D)$, where $D \in \mathbb{R}^{+}$refers to the diversity of $\beta$ values ( $\beta$-Diversity). The higher the value of $D$, the larger the diversity in the criticality values $\beta$ of the buyers.

Figure 4 illustrates the performance (social value) of each mechanism with regards to $D$ ( $\beta$-Diversity). For $D \approx 0$ (buyers approximate the neutral behavior, $\beta \approx 0$ ), all

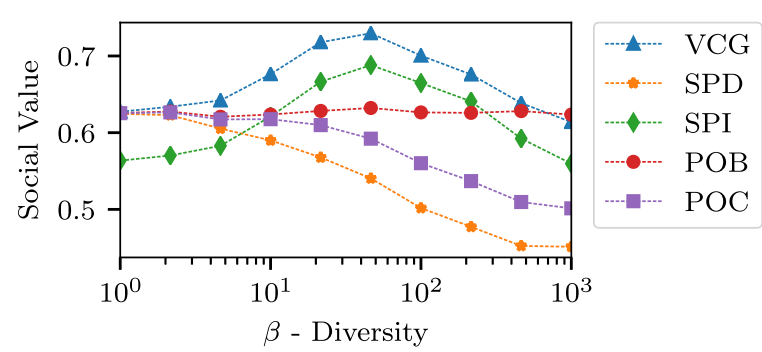

Fig. 4 Diversity in the criticality. Social value with regards to the diversity of criticality, for very low values of $\beta$-diversity all buyers approximate neutral attitude towards uncertainty in the delivery. For higher values of $\beta$-diversity buyers have increasingly varying criticality 
mechanisms apart from SPI achieve similar performance. By auctioning off SLAs of increasing reliability to the buyers (starting from the lowest reliability) the likelihood to obtain higher values from buyers assigned SLAs early (and at low reliability) decreases. For $D \in\left[10^{1}, 10^{2}\right]$, there is a clear distinction between the performance different mechanisms achieve, where VCG mechanism obtains its maximum performance. VCG yields higher social value than all other mechanisms for almost the whole range of $D$. In the same range, SPI shows a significant increase in social value by prioritizing over tolerant buyers in the allocation ${ }^{1}$. The opposite is observed for SPD, which is the result of auctioning SLAs of decreasing reliability (See "Vickrey-Clarke-Groves mechanism" section). For $D \approx 10^{3}$, the performance of all mechanisms decreases below the performance of the baseline POB, which is not affected by the increasing diversity of $\beta$ (neutral buyers). For high diversity the probability of extremely critical buyers is increased. Consequently, the average social value is decreasing. In settings where buyers demonstrate extreme behavior with regards to the criticality $(\beta \ll 0)$ the efficiency of the system is vastly affected. We showed how diversity in criticality $\beta$ affects the social value achieved by the studied mechanisms, as well as the large improvement in the social value that VCG achieves.

\section{Demand over supply ratio}

In this section we show that even in the case of large variations in the private value $\alpha$, VCG mechanism achieves an advantage in social value over the rest of the studied mechanisms. We use a diverse set of $\alpha, \forall i \in \mathcal{B}, \alpha_{i} \sim \mathcal{U}(0.1,1.0)$, which captures the highly irregular private values of dissimilar buyers in electricity systems. In addition, we use small $\beta$-diversity, $\beta_{i} \sim \mathcal{U}(-5,+5)$, for buyers that do not exhibit extreme tolerant or critical behaviors. Figure 5 (top) illustrates the social value with regards to the ratio of the total demand over the expected supply. In the case where all the demand is served with high probability (ratio $<0.5$ ), there is no significant difference between the different allocation
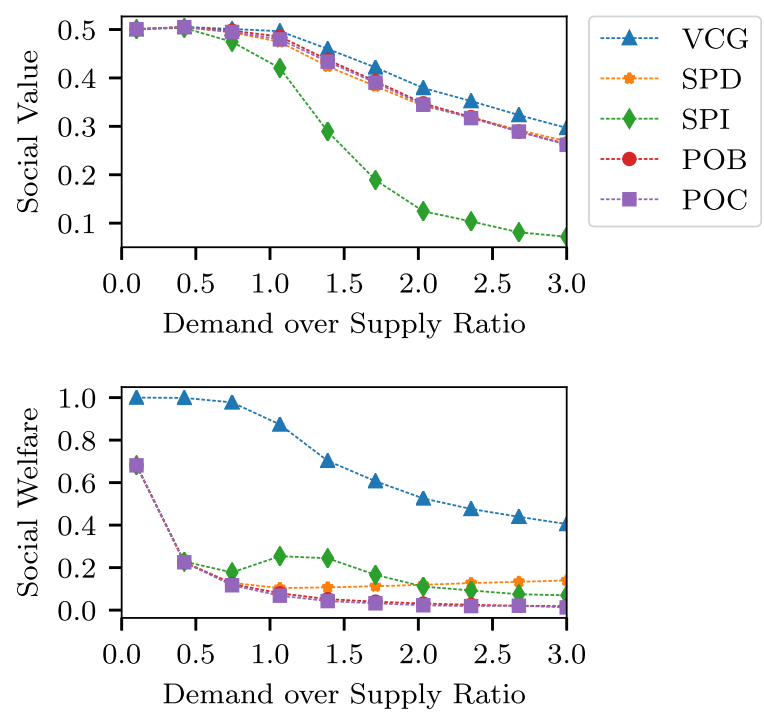

Fig. 5 Social value and welfare. Social value (top) and social welfare (bottom) achieved by different allocation mechanisms, namely, VCG, SSPA with decreasing reliability sequential auctions (SPD), increasing reliability sequential auctions (SPI), and two variants of a baseline method, POB and POC. The social value is computed as the average of all buyers' values with regards to the ratio of demand over the expected supply. Social welfare is normalized with respect to the social value obtained by the optimal VCG allocation 
mechanisms with respect to the social value. As the ratio increases it naturally follows that social value is decreasing for all allocation mechanisms. We can observe that the social value obtained by VCG is higher than any other allocation mechanism, and that there is a drop in the performance of SPI for demand to supply ratio higher than 1 . The performance of the remaining methods does not vary significantly. VCG achieves the highest social value even in the case where $\alpha$ varies significantly among the buyers.

\section{Social value $\&$ social welfare}

Finally, we show that the social welfare under VCG mechanism approximates the social value when the total demand is lower or approximately equal to the expected supply. Figure 5 (bottom) presents the social welfare achieved by all evaluated mechanisms, with respect to the ratio of the total demand over the expected supply. The social welfare is normalized with regards to the social value obtained by VCG mechanism (under the optimal allocation), ( $\left.S W / S V_{V C G}\right)$. The normalized social welfare is equal to the ratio of social value remaining to the buyers, while the rest is transferred to the mechanism (seller) through payments. Up to ratio $\approx 1$, the social welfare achieved by VCG mechanism is minimum at the $90 \%$ of the social value obtained using the optimal VCG allocation. The increased social welfare under SPI mechanism for ratio $>0.5$ is a natural consequence, since more low-reliability-SLAs become available when the ratio increases ${ }^{1}$. On the contrary, SPD achieves around 15\% of the optimal social value, however it exhibits a more stable (although lower) social welfare than SPI. The social welfare under the baseline mechanisms POB, POC approximates zero for high values of demand to expected supply ratio, and consequently most of the social value is transferred through payments to the mechanism.

In this section, we evaluated and presented the performance of the discussed mechanisms in terms of the social value and social welfare, for different settings. The VCG mechanism allocates SLAs in a social optimally manner and consequently the social value and welfare of the system is maximized for this mechanism. Sequential mechanisms such as SPD and SPI achieve lower social value and welfare than VCG, however they have an advantage over baseline allocations using only the VoLL. Additionally, they are easy to be used in practice without any computational limitations.

\section{Conclusion}

We proposed a contracting framework and mechanisms to allocate SLAs for electricity trading under uncertain supply and varying demand criticality of the buyers. We adapted SLAs as a direct extension of current conventional tariffs for use in electricity markets under uncertainty, and we defined the set of features that SLAs comprise, quantity, reliability, and price (see "Service level agreements" section). We further defined a generalized value function for buyers with regards to the criticality of their demand in the face of uncertain delivery (see "Critical \& tolerant buyers" section). The proposed value function generalizes the concept of the value of lost load (VoLL) with regards to the risk of unsuccessful delivery. The allocation of the SLAs to varying types of buyers as it results from two Vickrey-based mechanisms: sequential second-price auction and VCG (see "Auction-based SLA allocation" section). Both mechanisms ensure that no buyer has an incentive to misreport its value under certain conditions. Last, we evaluated the two mechanisms in an experimental study showing that VCG performance dominates all 
other allocations over a wide range of settings, and vastly improves the efficiency of the proposed system when compared to baseline allocation mechanisms considering only the VoLL (see "Evaluation \& discussion" section).

This work enables distributed electricity trading under uncertainty, and may also serve as a broad basis for future extensions: (i) In this paper we have considered no agency for the seller, the study of the seller incentives to misreport its reliability function can therefore be a direct extension. (ii) The exact characterization of the values functions of the buyers participating in the proposed electricity market under the presence of uncertain delivery. (iii) The enrichment of the features included in the SLAs e.g., time, penalties for misreport, no-delivery penalty, (iv) the multi-unit demand case, (v) the presence of an outside option for the buyers, e.g., multiple sellers, and last (vi), evaluation of the proposed mechanisms in real-world scenarios, e.g., in a microgrid with available renewable supply and heat pumps that act as buyers for whom temperature variations introduce heterogeneous criticalities. In view of the attained properties and performance, we believe that using SLAs as we delineated here provides a promising avenue for addressing electricity trading in the future smart grid. In particular, VCG allocation of SLAs is computable in $O\left(n^{3}\right)$, making it viable to assign the risk of demand curtailment to buyers that perform this task with low social cost. It can therefore be a tractable solution for peer-to-peer trading to balance local fluctuations in islanded grid scenarios or microgrids.

\section{Endnote}

${ }^{1}$ In SPI it is more likely that tolerant buyers are assigned low reliability SLAs, since their value for low reliability is higher than other types of buyers.

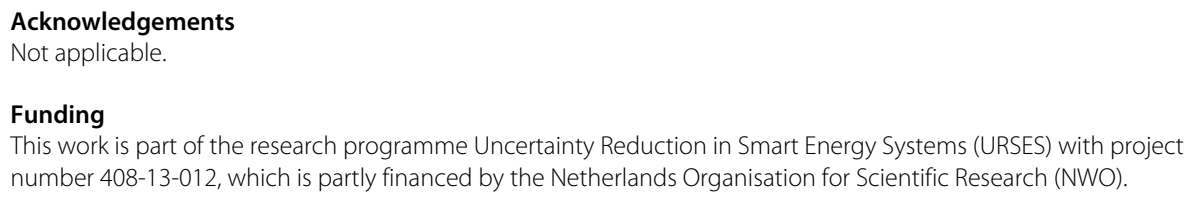

\section{Publisher's Note}

Springer Nature remains neutral with regard to jurisdictional claims in published maps and institutional affiliations.

Received: 24 May 2018 Accepted: 10 October 2018

Published online: 08 November 2018

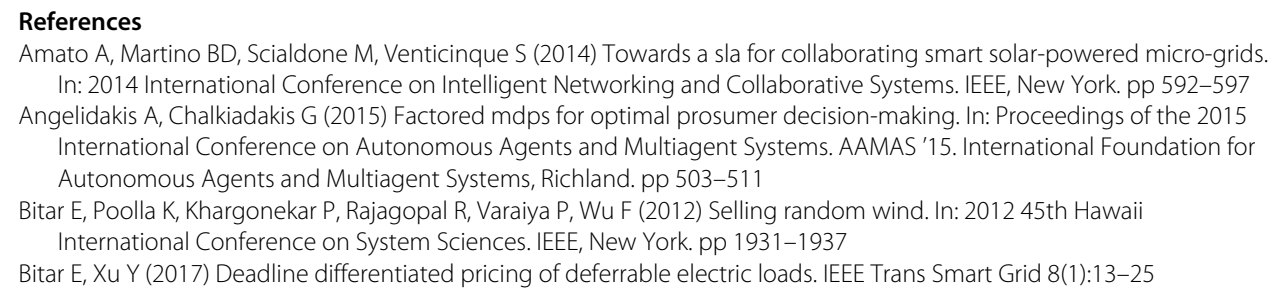


Braithwait S, Hansen D, O'Sheasy M (2007) Retail electricity pricing and rate design in evolving markets. Edison Electric Institute, Washington

Chao H-p, Oren SS, Smith SA, Wilson RB (1986) Multilevel demand subscription pricing for electric power. Energy Econ 8(4):199-217

Contreras J, Candiles O, de la Fuente Jl, Gomez T (2001) Auction design in day-ahead electricity markets. IEEE Trans Power Syst 16(3):409-417

Cramton P, Shoham Y, Steinberg R (2007) An overview of combinatorial auctions. SIGecom Exch 7(1):3-14

Dash RK, Vytelingum P, Rogers A, David E, Jennings NR (2007) Market-based task allocation mechanisms for limited-capacity suppliers. IEEE Trans Syst Man Cybern Part A Syst Hum 37(3):391-405

Date K, Nagi R (2016) Gpu-accelerated hungarian algorithms for the linear assignment problem. Parallel Comput $57(\mathrm{C}): 52-72$

Elkind E, Fatima S (2007) Maximizing revenue in sequential auctions. In: Internet and Network Economics. Springer Berlin Heidelberg, Berlin. pp 491-502

Farhangi $\mathrm{H}$ (2010) The path of the smart grid. IEEE Power Energy Mag 8(1):18-28

Gerding EH, Stein S, Ceppi S, Robu V (2016) Online mechanism design for vehicle-to-grid car parks. In: Proceedings of the Twenty-Fifth International Joint Conference on Artificial Intelligence. IJCAl'16. AAAI Press, Palo Alto. pp 286-293

Gustavsson R, Hussain S, Nordström L (2011) Engineering of trustworthy smart grids implementing service level agreements. In: Intelligent System Application to Power Systems (ISAP), 2011 16th International Conference On. IEEE, New York. pp 1-6

Hurwicz L, Reiter S (2006) Designing Economic Mechanisms. Cambridge University Press, Cambridge

Hussain S, Gustavsson R, Saleem A, Nordström L (2012) Service level agreement: Coordination and monitoring of actors in smart grid. In: Innovative Smart Grid Technologies (ISGT Europe), 2012 3rd IEEE PES International Conference and Exhibition On. IEEE, New York. pp 1-7

Ingersoll JE (1987) Theory of Financial Decision Making, vol. 3. Rowman \& Littlefield, Lanham

Krishna V (2010) Chapter two - private value auctions: A first look. In: Krishna V (ed). Auction Theory (Second Edition), Second edition. Academic Press, San Diego. pp 11-26

Kuhn HW (1955) The hungarian method for the assignment problem. Nav Res Logist Q 2(1-2):83-97

Kariuki KK, Allan RN (1996) Evaluation of reliability worth and value of lost load. IEE Proc Gener Transm Distrib 143(2):171-180

Leme RP, Syrgkanis V, Tardos E (2012) Sequential auctions and externalities. In: Proceedings of the Twenty-third Annual ACM-SIAM Symposium on Discrete Algorithms, SODA '12. Society for Industrial and Applied Mathematics, Philadelphia. pp 869-886

Ma H, Robu V, Li N, Parkes DC (2016) Incentivizing reliability in demand-side response. In: Proceedings of the Twenty-Fifth International Joint Conference on Artificial Intelligence. IJCAl'16. AAAI Press, Palo Alto. pp 352-358

Methenitis G, Kaisers M, La Poutré H (2017) Sla-mechanisms for electricity trading under volatile supply and varying criticality of demand. In: Proceedings of the 16th Conference on Autonomous Agents and MultiAgent Systems. International Foundation for Autonomous Agents and Multiagent Systems, Richland. pp 1640-1642

Methenitis G, Kaisers M, Poutré HL (2016) Incentivizing intelligent customer behavior in smart-grids: A risk-sharing tariff \& optimal strategies. In: Proceedings of the Twenty-Fifth International Joint Conference on Artificial Intelligence. IJCAI'16. AAAI Press, Palo Alto. pp 380-386

Neely MJ, Tehrani AS, Dimakis AG (2010) Efficient algorithms for renewable energy allocation to delay tolerant consumers. In: 2010 First IEEE International Conference on Smart Grid Communications. IEEE, New York. pp 549-554

Nisan N, Ronen A (2001) Algorithmic mechanism design. Game Econ Behav 35(1):166-196

Nisan N, Roughgarden T, Tardos E, Vazirani W (2007) Algorithmic Game Theory, vol. 1. Cambridge University Press, Cambridge

Oren SS, Smith SA (1993) Service Opportunities for Electric Utilities: Creating Differentiated Products: Creating Differentiated Products, vol. 13. Springer, New York

Palensky P, Dietrich D (2011) Demand side management: Demand response, intelligent energy systems, and smart loads. IEEE Trans Ind Inform 7(3):381-388

Salah F, Flath CM, Schuller A, Will C, Weinhardt C (2017) Morphological analysis of energy services: Paving the way to quality differentiation in the power sector. Energy Policy 106:614-624

Siddiqi SN, Baughman ML (1995) Reliability differentiated pricing of spinning reserve. IEEE Trans Power Syst 10(3):1211-1218

Silaghi GC, Şerban LD, Litan CM (2012) A time-constrained \{SLA\} negotiation strategy in competitive computational grids. Futur Gener Comput Syst 28(8):1303-1315

Strbac G (2008) Demand side management: Benefits and challenges. Energy Pol 36(12):4419-4426

Ströhle P, Flath CM (2016) Local matching of flexible load in smart grids. Eur J Oper Res 253(3):811-824

Ströhle P, Gerding EH, de Weerdt MM, Stein S, Robu V (2014) Online mechanism design for scheduling non-preemptive jobs under uncertain supply and demand. In: Proceedings of the 2014 International Conference on Autonomous Agents and Multi-agent Systems. AAMAS '14. International Foundation for Autonomous Agents and Multiagent Systems, Richland. pp 437-444

Syrgkanis V, Tardos E (2012) Bayesian sequential auctions. In: Proceedings of the 13th ACM Conference on Electronic Commerce, EC '12. ACM, New York. pp 929-944

Verma D (1999) Supporting Service Level Agreements on IP Networks. Macmillan Technical Publishing, London Vickrey W (1961) Counterspeculation, auctions, and competitive sealed tenders. J Finan 16(1):8-37

Vinyals M, Robu V, Rogers A, Jennings NR (2014) Prediction-of-use games: A cooperative game theoryapproach to sustainable energy tariffs. In: Proceedings of the 2014 International Conference on Autonomous Agents and Multi-agent Systems. AAMAS '14. International Foundation for Autonomous Agents and Multiagent Systems, Richland. pp 829-836

Walraven E, Spaan MTJ (2015) Planning under uncertainty with weighted state scenarios. In: Proceedings of the Thirty-First Conference on Uncertainty in Artificial Intelligence. UAl'15. AUAI Press, Arlington. pp 912-921 